\title{
The Corner Detection of Image based on Harris Algorithm
}

\author{
Chen Rong ${ }^{1, \text { a }}$ \\ ${ }^{1}$ Nanchang Normal University,Nanchang,330032,China \\ aemail: xinsea@163.com
}

Keywords: Image Processing;Corner Detection;Harris Algorithm;Feature;Threshold

\begin{abstract}
Image matching is a key technology to be solved in the fields of digital image processing and computer vision.Matching based on feature points is most widely used now.How to locate the right feature points is a vital issue.Only accurate feature points can lead right matching results. The corner is one of the important features of images. Corner detection plays an important role in image processing and computer vision,which is a kind of important feature extraction method in image processing.In order to improve the accuracy of corner detection in image,and remove noise interference effectively,the paper introduces a method of corner detection based on Harris algorithm, which are described briefly the basic principle and basic implementation process.The experiment shows that this algorithm is simple and easy to operate,the corner extracted are very effective.This method makes the precision and efficiency of image registration improved greatly.
\end{abstract}

\section{Introduction}

Image features include gray feature, texture feature, color feature, corner feature, regional feature, edge feature and contour feature, the corner and edge features are the most commonly used.The methods of corner detection generally can be divided into two categories,one category is the detection method based on image edge,to determine the corner point by the features of image edge, such as Prewitt operator, Sobel operator, Canny operator and Laplacian operator. Another category is the detection method based on image gray value, to determine the corner point to calculate gray image by local maximum curvature, such as Moravec operator, Harris operator and Susan operator. Corner detection base on Harris algorithm have simple operation and less sensitive to noise, which is widely used.

\section{The definition of Corner}

The corner is the larger point of gray change by a local window extending in all directions of mobile[1],corner is a point which has a tiny change will have a significant impact on some property of the image[2].Corner is the point which has the dramatic change of the gray and gradient for image, also has very high information content. The corner is the most important form of feature point,the more accurate corner is in understanding, the more contribute to the understanding and analysis of the image.Corners are defined as follows, the point is a local maximum of the derivative of pixel, is the intersection of the edge, is discontinuous at the edge of the object, is the large for the rate of change gradient and gradient direction of the point. A gradient operator reflects changes in any direction on the gray intensity of pixels which can effectively distinguish the corner and edge pixel[3].

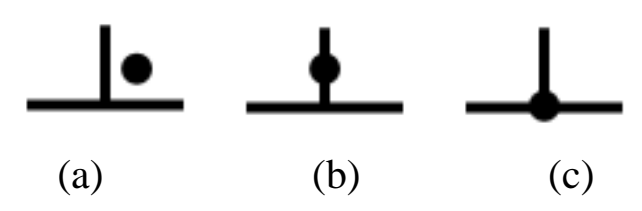

Fig.1. Point type(a) Not boundary point (b) Boundary point(c) Corner 


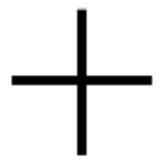

(a) $n=1$

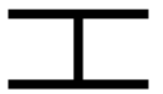

(b) $n=2$

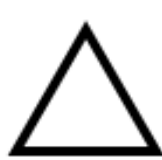

(c) $n=3$

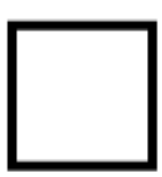

(d) $n=4$

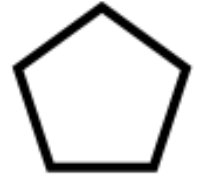

(e) $n=5$

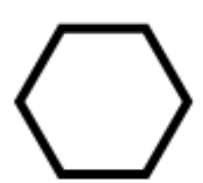

(f) $n=6$

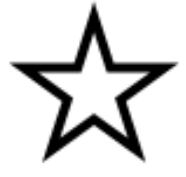

(g) $n=10$

Fig.2. the number of corner

\section{Corner detection based on Harris}

The main idea of corner detection algorithm based on Harris is that tiny moving along all directions in the window by using a small local test window around each pixel, computing the average changes of gray level. If the change value of intensity is greater than preset the threshold, the center pixel in the window is corner[4-5]. Corner detection methods are including Moravec, SUSAN, Harris algorithm. The corner detection based on Moravec algorithm is simple, but the calculated amount is too big, slow running, the false detection rate is very high, prone to a lot of false corners. Corner detection based on SUSAN algorithm is easy to locate good corner, and will produce lots of the corner in the area, it is difficult to find the good rules to remove these corner[6]. Corner detection based on Harris algorithm is proposed the feature corner extraction operator by Harris and Stephens in 1988[7].It uses the Taylor series expansion, the calculates gray value after window moving along arbitrary direction, and then calculates the feature points by the method of mathematics[8]. The Harris algorithm is improved based on Moravec operator, and then has formed the corner detection based on Harris algorithm. The Harris operator can be obtained autocorrelation changes from all directions, it uses a autocorrelation function to calculate the gray signals which have obvious changes in the two-dimensional direction of pixel position, structure of a related matrix $\mathrm{M}$, by comparing the feature value of the matrix $\mathrm{M}$ can easily extract the corner points of the corresponding. Constructing a matrix $\mathrm{M}$ associated with it, can easily extract the corresponding corner by comparing the feature value of the matrix M[9], definition function is:

$$
\begin{aligned}
& M=\sum_{x, y}\left[w(x, y)\left(\begin{array}{cc}
I_{x}^{2} & I_{x} I_{y} \\
I_{x} I_{y} & I_{y}{ }^{2}
\end{array}\right)\right] \\
& w(x, y)=\frac{1}{2 \pi \sigma^{2}} e^{-\left(x^{2}+y^{2}\right) / 2 \sigma^{2}}
\end{aligned}
$$

In the formula, $w(x, y)$ is for the Gauss function, ${ }^{x}$ is a gradient for $\mathrm{x}$ direction, ${ }^{{ }}{ }_{\text {is a }}$ gradient for y direction.

After diagonalization transform, $\mathrm{M}$ can be expressed as follows.

$$
M=P^{-1}\left[\begin{array}{ll}
\lambda_{1} & 0 \\
0 & \lambda_{2}
\end{array}\right] P
$$

In the formula, $\lambda_{1}$ and $\lambda_{2}$ is for the two eigenvalue of the M matrix.

The response function of corner detection based on Harris algorithm is shown in equation.

$$
C R F=\operatorname{det}(\mathrm{M})-\mathrm{K}^{*}(\operatorname{Tr}(\mathrm{M}))^{2}
$$

In the formula, $\operatorname{det}(M)$ is the determinant of the matrix $M, \operatorname{Tr}(M)$ is the trace of matrix $M, K$ is a constant, $\mathrm{K}$ is the experience value.

The eigenvalues of matrix $\mathrm{M}$ is a first-order curvature of autocorrelation function,and the eigenvalues of matrix $\mathrm{M}$ is associated with the properties of feature points. If the two eigenvalues are relatively small,the point is in a flat area, is not a boundary point or corner. If the eigenvalues have a value is relatively small, and another value is relatively large,the point is 
the boundary point. If the two eigenvalues are large, moving along any direction will cause dramatic changes in gray,then the point is the corner. This curvature in any direction of point are larger, the corner to be extracted.

\section{The effect of corner detection based on Harris}

The detection process based on Harris algorithm is as follows.

(a) To make use of differential operators for image filtering,and calculate $I_{x}{ }^{2}, I_{y}{ }^{2}, I_{x} I_{y}$ for each pixel in the image.

(b) In order to remove the noise,Gauss smooth on $I_{x}{ }^{2}, I_{y}{ }^{2}, I_{x} I_{y}$.In the process, the normalized template parameter is set to 1 .

(c) To determine the local maxima by non maxima suppression,non maximum suppression has two main steps, first use algorithm to detect real corner according to the set threshold, and then to record the real coordinates of these corner[10].

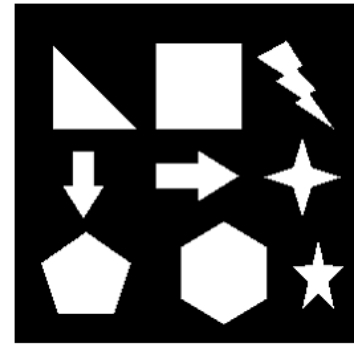

(a) Origin image

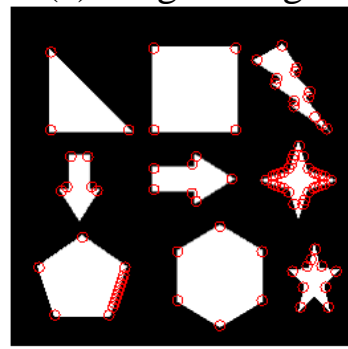

(e) $\mathrm{K}=0.07$

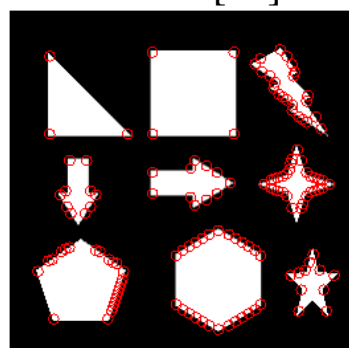

(b) $\mathrm{K}=0.04$

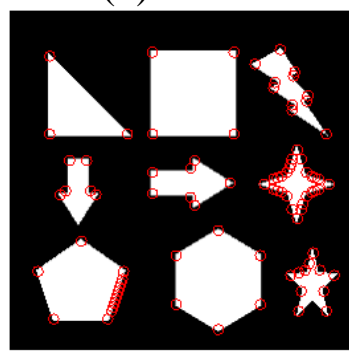

(f) $\mathrm{K}=0.08$

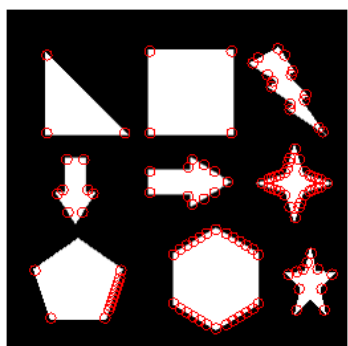

(c) $\mathrm{K}=0.05$

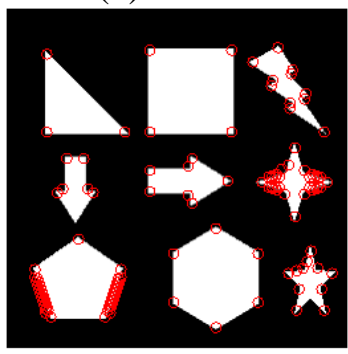

(g) $\mathrm{K}=0.10$

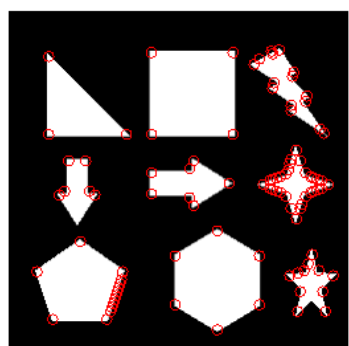

(d) $\mathrm{K}=0.06$

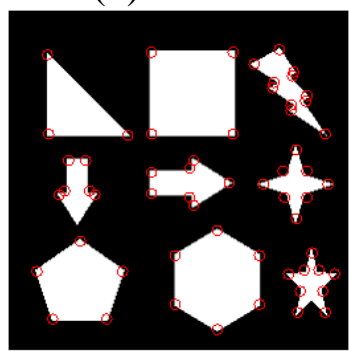

(h) $\mathrm{K}=0.11$

Fig.3. Corner detection based on Harris.

Table 1. Relationship between the value of constant $\mathrm{K}$ in corner response function and the number of corner

\begin{tabular}{cccc}
\hline $\mathrm{K}$ & Count & $\mathrm{K}$ & Count \\
\hline 0.04 & 141 & 0.05 & 122 \\
0.06 & 97 & 0.07 & 93 \\
0.08 & 89 & 0.09 & 86 \\
0.10 & 124 & 0.11 & 59 \\
0.12 & 59 & 0.13 & 56 \\
0.14 & 61 & 0.15 & 53 \\
\hline
\end{tabular}

\section{Conclusion}

Corner detection based on Harris algorithm is a classical corner detection algorithm, which has rotation and affine invariance, which is a derivative, fast running speed, feature extraction is reliable, has certain stability and robustness.Corner detection is completely dependent on the threshold setting.From the experimental results, the larger threshold becomes, the less the number of corners have, the registration reliability rise, the number of corner decreases more slowly and gradually stabilized. 


\section{Acknowledgement}

In this paper, the research was sponsored by the key discipline of Nanchang Normal University,China (Project No.NSXK20141001) and the Reform in Education of Nanchang Normal University,China (Project No.JGKT-14-18).

\section{References}

[1] LI Mang.Image tamper detection based on Harris corner and quality assessment[J].Electronic Design Engineering,22(17):189-193

[2]Jian Li,Xueli Xu,Xueyuan Lan,Chengyan Zhang.Feature Extraction and Measurement of the Human Body Based on Harris Corner Detection[J].Computer Measurement \& Control,22(2):367-369

[3]YANG Hui,YANG Hui-cheng,WANG Xiao-weiqian. Parts Shape Recognition Based on Improved Harris Corner Detection Algorithm[J].Journal of Chongqing University of Technology( Natural Science),27(12):64-67

[4] Zhang Jing,Li Yu-long,Wang Yang-ping.An Improved Harris Corner Detection Algorithm Based on B spline Function[J]. Journal of Lanzhou Jiaotong University,32(6):32-35

[5]GE Pan-pan,CHEN Qiang,GU Yi-he.Algorithm of remote sensing image matching based on Harris corner and surf feature[J].Application Research of Computers,31(7):2205-2208

[6]WU Yan,CHEN Zhong-jin,YUE Zhi-wen.Vehicle license plate location based on Harris corner and AP clustering[J].Guangxi University of Science and Technology, 25(2):54-57

[7]Zhang Cong-peng,Wei Xue-guang.Rectangle detection based on Harris corner[J].Optics and Precision Engineering,22(8):2259-2266

[8]YANG Chen-chen,GU Guo-hua,QIAN Wei-xian,CHEN Qian,XU Fu-yuan.Hardware Implementation of Infrared Image Registration Based on the Harris Corner of KLT Tracking[J]. Infrared Technology,35(10):632-637

[9] Li Pengcheng,Zeng Yumin,Zhang Meng. Improved Image Mosaic Algorithm Based on Harris Corner[J]. Journal of Nan Jing Normal University (Engineering and Technology Edition), 14(1):70-75

[10] Lv Jing-guo,Yang De-he,Wang Qing-song.Fast 3-D Feature Point Detector Based on Harris [J].Remote Sensing Information,28(5):18-20 